\title{
A negative result in differentiation theory
}

by

BERNARDO LÓPEZ MELERO (Madrid)

Abstract. Given a differentiation basis in $\boldsymbol{R}^{m}$, we construct counterexamples to a.e. differentiability which remain counterexamples after any rotation of the basis, and we prove that they are typical elements (in the sense of Baire's category) of the Orlicz spaces to which they belong.

Notation. A Busemann-Feller differentiation basis $\mathscr{B}$ is a family of open subsets of $\boldsymbol{R}^{m}$ with every $x \in \boldsymbol{R}^{m}$ belonging to sets of arbitrarily small diameter which are in $\mathscr{B} .|A|$ will denote the Lebesgue measure, and we refer to

$$
D \int f(x)=\lim \left\{(1 /|R|) \int_{R} f, x \in R \in \mathscr{B}\right\}
$$

and to

$$
\bar{D} \int f(x)=\limsup \left\{(1 /|R|) \int_{R} f, x \in R \in \mathscr{B}\right\}
$$

as the derivative and the upper derivative of $\int f$ relative to $\mathscr{B}$ at the point $x$, the lim's being taken as the diameter of $R$ decreases to 0 . $M$ will be the maximal operator associated with $\mathscr{B}$ :

$$
M f(x)=\sup \left\{(1 /|R|) \int_{R}|f|, x \in R \in \mathscr{B}\right\} .
$$

$C$ will be some positive constant, not always the same.

Introduction. It is a well known fact that if we substitute $n$-dimensional intervals for the balls or cubes in Lebesgue's differentiation theorem, it turns out to be false for some $f \in L^{1}$, and oven in one sense (Baire's category) for almost every $f \in L^{1}$ at every point of $\boldsymbol{R}^{m}$ (Saks' xarity theorem, see [3]). To see the relevance of this fact, let us suppose for instance that we are dealing with the rectangular Cesáro means of a function $f \in L^{1}\left(\boldsymbol{R}^{2}\right)$ at the point $\left(x_{0}, y_{0}\right)$ :

$$
f_{R, S}\left(x_{0}, y_{0}\right)=\int f\left(x_{0}-x, y_{0}-y\right) \cdot K_{R}(x) K_{S}(y) d x d y,
$$

where $K_{R}(x)=\left(1 / 2 \pi^{2} R\right)\left((1-\cos (2 \pi R x)) / x^{2}\right)$ is the Fejér kernel. 
We have $\lim _{R \rightarrow \infty} f_{R_{2} R}\left(x_{0}, y_{0}\right)=f\left(x_{0}, y_{0}\right)$ for a.e. $\left(x_{0}, y_{0}\right)$, but we cannot $\underset{R \rightarrow \infty}{R e}$ the same for the unrestricted $R, S \rightarrow \infty$ limit because expect to have the same for the untests the mean of $f$ over the interval $(-1 / R, 1 / R) \times(-1 / S, 1 / S)$ and the existence of the latter limit would imply Lebesgue's differentiation theorem for the interval basis.

Saks' rarity theorem is only an example of the following general situation: let $\mathscr{B}$ be a Busemann-Feller differentiation basis in $\boldsymbol{H}^{m}$ that is homothecy invariant and let $\Phi$ be its halo function, i.e.,

$$
\Phi(u)=\sup \left\{\left|\left\{M_{\chi_{A}}>1 / u\right\}\right|, A \subset \boldsymbol{R}^{m},|A|=1\right\} .
$$

Then in every Orlicz class $\Psi(L)$ with $\Psi$ smaller than $\Phi$ at infinity, we can find some $f$ which is not differentiated by the basis (see [3]), and this implies that the same is true for every $f \in \Psi(L)$ except those in a set of the first category, as Moriyón [6] has proved.

If $\mathscr{B}$ is again the interval basis in $\boldsymbol{R}^{2}$, we know from the theorem of Jessen, Marcinkiewicz and Zygmund [4] that it differentiates overy $f \in L\left(\log ^{+} L\right)_{\text {loc }}$, so that $\Phi(u)$ cannot be greater at infinity than $u(\log u)$, and direct computation shows that it is exactly of the same order, so that we can substitute anything like $L\left(\log ^{+} L\right)^{1 / 2}$ or $L\left(\log ^{+} L\right) / \log ^{+}\left(\log ^{+} L\right)$ for $L^{1}$ in Saks' rarity theorem.

On the other hand, Zygmund had raised the following question, which may seem natural in view of the previous considerations: given $f \in L^{1}\left(\boldsymbol{R}^{2}\right)$, will it always be possible to tind a pair of rectangular axes such that the interval basis in those axes will differentiate $f$ ?

Marstrand [5] answered this in the negative, exhibiting an $f \in L^{1}$ such that : or every rotation of the axes the upper derivative of $f$ relative to the rotated interval basis is $+\infty$ almost everywhere, and this was

improved upon by El Helou [2], who found such an $f$ in $\bigcap_{a<1} L\left(\log ^{+} L\right)^{a}$.
Our purpose here is to show:

(a) that the idea in Marstrand's construction works with any translation invariant Busemann-Feller differentiation busis, and

(b) that things are, here also, as they are in Saks' rarity theorem.

These two ideas were suggested to me by Antonio Oordoba and Miguel de Guzmán, whom I am glad to thank hero.

Result. We suppose first that $\mathscr{B}$ is also homothecy invariant and define, for $0<r<1$,

$$
H(r)=\bigcup\left\{R \in \mathscr{B}, R \subset B_{1},\left|R \cap B_{r}\right| /|R|>r\right\}, \quad \tilde{\Phi}(u)=u^{m}|H(1 / u)|,
$$

where $B_{r}$ is the ball with radius $r$ around 0 . (For the definition in tho general case, see Remark 1.) that

THEOREM. Let $\Psi:(1, \infty) \rightarrow \boldsymbol{R}$ be an increasing, convex function such

$$
\lim _{u \rightarrow \infty}(\Psi(u) / \tilde{\Phi}(u))=0
$$

Then every $f \in \Psi(L)$ except those in a set of the first category in $\Psi(L)$ verifies that: for every rotation $\gamma$ of $\boldsymbol{R}^{m}$ the upper derivative of $\int f$ relative to the basis $\mathscr{B}_{\gamma}$ obtained by rotating $\mathscr{B}$ through $\gamma$, is $+\infty$ almost everywhere.

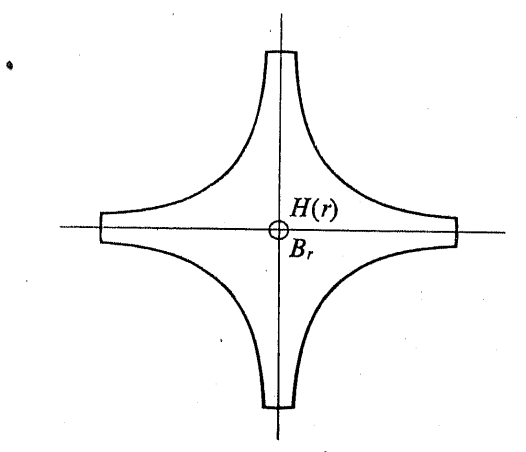

Fig. 1

We note that for the interval basis in $\boldsymbol{R}^{2}$ (see Fig. 1) an easy computation shows $\tilde{\Phi}(u) \simeq O u(\log u)$, which gives the result of [5] in the stronger version of [2] if we take for instance $\Psi(u)=u(\log u)(\log \log u)^{-1}$.

A small-scale model of the proof. We shall now use some of the ideas of the proof below to give a demonstration of this known fact: there is an $f \in I^{1}\left(\boldsymbol{R}^{2}\right)$ which is not differentiated by the interval basis. In fact, we shall construct $f \in L^{1}$ such that $\bar{D} \int f(x)=\infty$ on a set of positive measure.

For each $n$, wo divide the unit square into $n^{2}$ equal squares and consider the $n^{2}$ bulls with radius $n^{-1} r_{n}$ concentric with them. We denote the union of these balls by $\sigma_{n}$. Next we consider the images of $H\left(r_{n}\right)$ by the homothecies that apply the ball $B_{r_{n}}$ onto each of the balls in $C_{n}$, and we call $K_{n}$ their union.

Observe that $\left|O_{n}\right|=\left|B_{r_{n}}\right|$ and $\left|K_{n}\right|=\left|H\left(r_{n}\right)\right| \simeq r_{n} \log \left(1 / r_{n}\right)$.

Now we take $f_{n}=\lambda_{n} \chi_{c_{n}}$ and $f=\sup _{n}$ (the numbers $r_{n}, \lambda_{n}>0$ will be determined later). 
To get $f \in L^{1}\left(\boldsymbol{R}^{2}\right)$ it is enough to have

$$
\sum_{n} r_{n}^{2} \lambda_{n}<\infty
$$

By the definition of $H\left(r_{n}\right)$ and the homothecy invarianee of the interval basis, $K_{n}$ is a union of intervals $R$ with diametiex less than $n^{-1}$ and such that

$$
\lambda_{n} r_{n}<(1 /|R|) \int_{R} f_{n} \leqslant(1 /|R|) \int_{R} f
$$

so that if $x \in \limsup K_{n}$ and

$$
\lim _{n} \lambda_{n} r_{n}=\infty
$$

$x$ belongs to a contracting sequence of these intervals, and wo have $\bar{D} \int f(x)=\infty$. So, let us look at the measure $\mid$ limsup $K_{n} \mid$. As it stiands, it need not be greater than $\lim \left|K_{n}\right| \simeq \lim r_{n} \log \left(1 / r_{n}\right)$, which must bo 0 to fulfil (1), but if we "move" the $K_{n}$ to prevent their intersecting too much, that measure is likely to increase. In fact, an easy application of the Borel-Cantelli theorem tells us that if wo havo

$$
\sum_{n}\left|K_{n}\right|=\infty
$$

then for some $x_{n} \in \boldsymbol{R}^{2}$ we have

$$
\left|\limsup \left(x_{n}+K_{n}\right)\right|>0 .
$$

This means that we shall get our $f$ as the sup of the shifted $\left.f_{n}(x)-x_{n}\right)$ if we can find numbers $r_{n}, \lambda_{n}>0$ satisfying (1), (2) and (3). Wo simply verify that

$$
r_{n}=\left(n \log ^{2} n\right)^{-1}, \quad \lambda_{n}=n \log ^{5 / 2} n
$$

will do the work.

Proof of the theorem. Wo construct $f_{n}$ as in the provious section. except for the fact that we divide the unit cube of $\boldsymbol{R}^{m}$ not into $n^{m}$ lout into $M_{n}^{m}$ equal subcubes, where the integers $M_{n} \rightarrow \infty$ will bo dotornined later. Consequently, the balls in $O_{n}$ now have radins $M_{n}^{-1} r_{n}$, and wo havo as before $\left|O_{n}\right|=\left|B_{r_{n}}\right|$. Finally, wo fix a rotation $\gamma$ and considex the images of $\gamma\left(H\left(r_{n}\right)\right)$ to form the set $K_{n}(\gamma)$, and we still have $\left|K_{n}(\gamma)\right|=\left|H_{n}\left(r_{n}\right)\right|$.
If we put $f=\sup _{n} f_{n}$ and the $\lambda_{n}$ are increasing,

$$
\int \Psi(f)=\sum_{n} \int_{\sigma_{n}-\bigcup_{n<k} \sigma_{k}} \Psi\left(\lambda_{n}\right)<0 \sum_{n} r_{n}^{m} \Psi\left(\lambda_{n}\right),
$$

so that if we want $f \in \Psi(L)$ we only need.

$$
\sum_{n} r_{n}^{m} \Psi\left(\lambda_{n}\right)<\infty
$$

Next, by the same reasoning as before applied to $K_{n}(\gamma)$ and the basis $\mathscr{B}_{\gamma}$, we have $\bar{D}_{\gamma} \int f(x)=\infty$ for $x \in \lim \sup K_{n}(\gamma)$ provided we have

$$
\lim _{n} \lambda_{n} r_{n}=\infty \text {. }
$$

So we again want $\left|\lim \sup K_{n}(\gamma)\right|>0$. Note that the Borel-Cantelli theorem cannot help us now because the sets $K_{n}(\gamma)$ are different for each $\gamma$ and they are very "thin" sets as $n$ increases, so that the required shiftings $x_{n}$ will be a crazy function of $\gamma$. So we abandon random shifting as the mixing method and use the following idea, which is the key to the whole construction: if the $M_{n}$ increase quickly enough, the sets $K_{n}(\gamma)$ are probabilistically almost independent, i.e.,

$$
\left|K_{n} \cap K_{n+1}\right| \simeq\left|K_{n}\right|\left|K_{n+1}\right|
$$

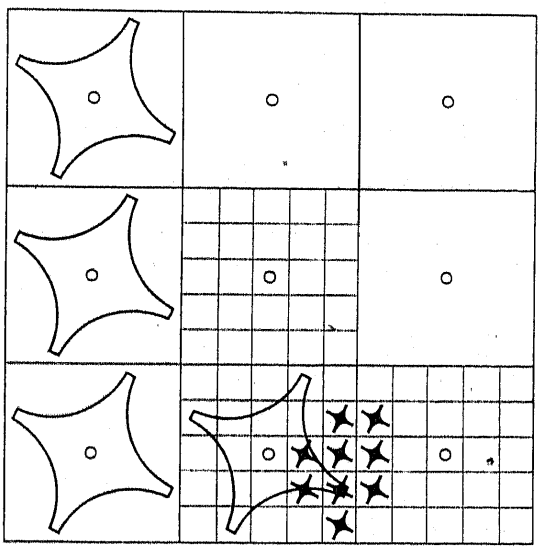

Fig. 2

(see Fig. 2) and the same is true for their complements, so that we obtain the following estimate (see Remark 2): 


$$
1-\left|\bigcup_{p \leqslant n \leqslant q} K_{n}(\gamma)\right|<\prod_{p \leqslant n \leqslant q}\left(1-(1 / 2)\left|H\left(r_{n}\right)\right|\right)
$$

But as

$$
\lim \sup K_{n}(\gamma)=\bigcap_{p} \bigcup_{n \geqslant p} K_{n}(\gamma)
$$

and

$$
1-\left|\lim \sup K_{n}(\gamma)\right| \leqslant \sum_{p}\left(1-\left|\bigcup_{p \leqslant n} K_{n}(\gamma)\right|\right),
$$

to get $\left|\limsup K_{n}(\gamma)\right|=1$ we only need, according to (E),

$$
\prod_{n}\left(1-(1 / 2)\left|H\left(r_{n}\right)\right|\right)=0
$$

or

$$
\sum_{n}\left|H\left(r_{n}\right)\right|=\infty
$$

We have arrived at the same three conditions as in the previous section and we have reduced our problem to that of finding numbers $r_{n}, \lambda_{n}$ satisfying (1), (2) and (3), and for this we apply to

$$
h(r)=|H(r)| \quad \text { and } \quad g(r)=r^{m} \Psi(1 / r)
$$

the following lemma, whose proof is easy:

LEMMA. Let $h, g:(0,1) \rightarrow \boldsymbol{R}$ such that

$$
\lim _{r \rightarrow 0} g(r)=\lim _{r \rightarrow 0}(g(r) / h(r))=0 .
$$

Then we can find two sequences $r_{n} \rightarrow 0, \lambda_{n} \rightarrow \infty$ such that

$$
\sum h\left(r_{n}\right)=\infty, \quad \sum\left(\lambda_{n} r_{n}\right)^{m} g\left(1 / \lambda_{n}\right)<\infty .
$$

We have now obtained a function that misbehares a.e. in the unit cube, and it is a trivial task to extend this to the whole $\boldsymbol{R}^{m}$ by adding a geometric series of multiples.

Only the "rarity" assertion in the theorem remains to be proved. For a given $f$ and $b, s>0$ consider the sét

$E(f, \gamma, b, s)=\left\{x \in\right.$ unit eube such that $(1 / \| R \mid) \int_{R} f<b$

$$
\text { if } \left.x \in R \in \mathscr{B}_{\gamma} \text { and } \operatorname{diam}(R)<s\right\} \text {. }
$$

Given $\varepsilon>0$, we are going to prowe that for the $f$ which we have constructed and a small enough $h \in \Psi(L)$ we have

$$
|E(f+h, \gamma, b, s)|<\varepsilon .
$$

We take $n_{0}$ such that $\forall n \geqslant n_{0} \lambda_{n} r_{n}>2 b$ and $M_{n}^{-1}<s$.
OLAIM. For more than half the points $y \in H(r)$ (in measure) there is some $\tilde{R} \in$ such that

$$
y \in \tilde{R}, \quad\left|\tilde{R} \cap B_{r}\right| /|\tilde{R}|>r
$$

and

$$
\left|\tilde{R} \cap B_{r}\right| \geqslant \alpha=\alpha(r)>0
$$

where $a$ depends on $\mathscr{B}$ and $r$ but not on $y$.

Let us then suppose that we took only that half of $H(r)$ as the whole $H(r)$ from the beginning (this does not modify our construction) and we take $h \in \Psi(L)$ such that $\|h\|_{1}<\lambda_{n} \alpha\left(r_{n}\right) M_{n}^{-m} / 2$. Then, for $x \in K_{n}(\gamma)$ and the bomothecy $\eta$ such that $x \in \eta \circ \gamma\left(H\left(r_{n}\right)\right)$, let $y=(\eta \circ \gamma)^{-1}(x), \tilde{R}$ as in the claim and $\boldsymbol{R}=(\eta \circ \gamma)(\tilde{\boldsymbol{R}})$. We have

$$
\begin{aligned}
(1 /|R|) \int_{R}(f+h) & \geqslant(1 /|R|)\left(\lambda_{n}\left|\eta \circ \gamma\left(\tilde{R} \cap B_{r_{n}}\right)\right|-\int_{R}|h|\right) \\
& >(1 / 2)(1 /|R|) \int_{R} f_{n}>\lambda_{n} r_{n} / 2,
\end{aligned}
$$

so that, if $n \geqslant n_{0},(1 /|R|) \int_{R}(f+h)>b$ and $\operatorname{diam}(R)<s$, i.e.,

$$
x \notin E(f+h, \gamma, b, s) .
$$

We now taike $n_{1} \geqslant n_{0}$ such that $\prod_{n_{0} \leqslant n \leqslant n_{1}}\left(1-(1 / 2)\left|H\left(r_{n}\right)\right|\right)<\varepsilon$, and this implies, as before:

$$
1-1 \bigcup_{n_{0} \leqslant n \leqslant n_{I}} K_{n}(\gamma) \mid<\varepsilon .
$$

As none of the preceding constants depended upon $\gamma$, we have proved that if $\|\hbar\|_{1}<\min _{n} \alpha\left(r_{n}\right) M_{n}^{-m} / 2$, then

$$
\forall \gamma|\mathbb{E}(f+h, \gamma, b, s)|<\boldsymbol{\iota} .
$$$$
n_{0} \leqslant n_{1} \leqslant n_{1}
$$

But $8, \mathbb{S}\|h\|_{1} \leqslant \sigma\|h\|_{\Psi(L)}$ (by the Jensen inequality), we have proved that $f$ is interior in the $\Psi(L)$ topology to each of the sets

$$
F_{b, s, s}=\{g \in \Psi(L), \forall \gamma|\mathbb{E}(g, \gamma, b, 8)|<\varepsilon\} .
$$

Given $g \in I^{\infty}$, we can repeat the same argument beginning with $\lambda_{n} r_{n}$ $>2\left(b+\|g\|_{\infty}\right)$, which gives $(1 /|R|) \int_{R}(f+h)>b+\|g\|_{\infty}$, i.e., $(1 /|R|) \int_{R}(f+$ $+g+h)>b$, so that the interior of $F_{b, s, s}$ contains $f+L^{\infty}$, which is dense in $\Psi(L)$.

It is now an easy exercise to prove that a function $f \in \Psi(L)$ is as bad as our theorem requires if and only if $f \in \bigcap_{b, 8,8} F_{b, 8,8}=\bigcap_{n} F_{n, 1 / n, 1 / n}$, which proves the theorem if we justify the claim. But this follows from 
$H(r)=\bigcup_{n} \bigcup\left\{R \in \mathscr{B}, R \subset B_{1},\left|R \cap B_{r}\right| /|R|>r\right.$ and $\left.|R|>1 / n\right\}$.

A trivial corollary of the rarity assertion in the theorem is that the typical $f \in \Psi(L)$ is doubly as bad as the one we have constructed: it verifies $\underline{D}_{\gamma} \int f(x)=-\infty$ as well.

\section{Examples and remarks.}

1. If $\mathscr{B}$ is not homothecy invariant, we define

$$
H(r, s)=\bigcup\left\{R \in \mathscr{B}, R \subset B_{s},\left|R \cap B_{r s}\right| /|R|>r\right\}, \quad 0<r, s<1
$$

and we build $K_{n}(\gamma)$ with translated, rotated copies of $H\left(r_{n}, M_{n}^{-1}\right)$. If we now define $h(r)=\underset{s \rightarrow 0}{\liminf }|H(r, s)| s^{-m}$, we still have the estimate

$$
1-\left|\bigcup_{p \leqslant q \leqslant n} K_{q}(\gamma)\right|<\prod_{p \leqslant q \leqslant n}\left(1-(1 / 2) h\left(r_{q}\right)\right)
$$

(which reduces to $(\mathrm{E})$ if $\mathscr{B}$ is homothecy invariant) and the theorem remains true with $\tilde{\Phi}(u)=u^{m} h(1 / u)$.

2. The validity of our estimate (E), which we entrusted in the proof to the geometric evidence of Fig. 2, can be justified by the following lemma:

LEMCMA. Suppose $E_{k}$ are measurable subsets of the unit oube $Q$ of $\boldsymbol{R}^{m}$ such that for every suboube $Q^{\prime}$ in the $j$-th dyadio subdivision of $Q$ we have, if $k>j$,

$$
\left|E_{k} \cap Q^{\prime}\right|=\left|E_{k}\right|\left|Q^{\prime}\right|
$$

Let $\left\{A_{a}\right\}$ be a family of measurable sets suoh that for $\varepsilon>0$ there are a compact $K_{\alpha}$ and an open $V_{\alpha}$ verifying $K_{\alpha} \subset A_{\alpha} \subset V_{\alpha},\left|V_{\alpha}-K_{\alpha}\right|<\varepsilon$ and $\operatorname{dist}\left(K_{a}, \nabla_{a}^{c}\right) \geqslant d(\varepsilon)>0$ uniformly in $\alpha$.

Then we have

$$
\lim _{k \rightarrow \infty}\left|A_{\alpha} \cap D_{k}\right|-\left|A_{\alpha}\right|\left|E_{k}\right|=0
$$

uniformly in $\alpha$.

We apply the lemma to get $\left(\mathrm{E}^{\prime}\right)$. Suppose we have chosen $M_{1}, \ldots$ $\ldots, M_{n-1} \cdot\left\{A_{\alpha}\right\}$ will be the sets $\bigcap_{p \leqslant q \leqslant n-1}\left(Q-K_{\alpha}(\gamma)\right)$ for all $\gamma$ and all $p=1, \ldots$ $\ldots, n-1$.

$E_{k}$ will be the complement of the union of $2^{\mathrm{km}}$ rotated, translated copies of $H\left(r_{n}, 2^{-k}\right)$, i.e., the candidate to be $Q-K_{n}(\gamma)$ if we choose
$M_{n}=2^{\gamma_{k}}$. Wo have for all $p, \gamma$ :

$$
\begin{aligned}
\left|\bigcap_{p \leqslant q \leqslant n}\left(Q-K_{\alpha}(\gamma)\right)\right| / & \prod_{p<q<n-1}\left(1-(1 / 2) h\left(r_{q}\right)\right) \\
& <\left(1-2^{k m}\left|H\left(r_{n}, 2^{-t}\right)\right|\right)+h\left(r_{n}\right) / 4 \\
& <1-\left(h\left(r_{n}\right)-h\left(r_{n}\right) / 4\right)+h\left(r_{n}\right) / 4=1-(1 / 2) h\left(r_{n}\right) .
\end{aligned}
$$

The first inoquality is true for all $k>$ some $j$ because of the lemma and tho induction hypothesis; the second is true for some $k>j$ because of the definition of $h\left(r_{n}\right)$. Thus we may take $M_{n}=2^{k}$ for this $k$.

3. Wo have computed $\Phi(u)$ for some bases that are only translation invariant; they ure of the type "intervals with dimensions $s_{1} \times \ldots \times s_{r} \times$ $\times p_{1}\left(s_{1}, \ldots, s_{r}\right) \times \ldots \times p_{m-r}\left(s_{1}, \ldots, s_{r}\right) "$, the $\varphi_{j}$ 's being increasing functions in each $s_{t}$; in the case of $r=2, m=3$, Obrdoba [1] has proved $L\left(\log ^{+} L\right)$ to be the border line, and the conjecture for the general case is $L\left(\log ^{+} L\right)^{r-1}$; for our theorom we also obtain $\tilde{\Phi}(u) \simeq O u(\log u)^{r-1}$ in the computed cases.

For the interval basis in $\boldsymbol{R}^{m}$ the result is also sharp: $\tilde{\Phi}(u)$ $\cong O u(\log u)^{m-1}$, which wo know to be as much as is possible from the JessenMarcinkiowicz-Zygmund theorem.

4. For the "rectangles in lacunary directions" basis, the computation was already made by Stromberg [8] to get a "lower bound conjecture". In fact, if $\Phi$ is the usual halo function and $\Phi_{1}(L)$ is the largest Orlicz class that differentiates, we obviously have at infinity $\tilde{\Phi}<\Phi<\Phi_{1}$.

For the $k$-generated set of exponential directions we have $\tilde{\Phi}(u)$ $\simeq O u(\log u)^{k+1}$ and so far nobody seems to know if $\Phi_{1} \simeq \tilde{\Phi}$ (the best result is $\Phi_{1}(u)<u^{p} \forall p>1$, by Nagel, Stein and Wainger [7]).

5. Wo know that the first inequality in Remark 4 is sometimes a strict ono: the "rectangles in all directions" basis gives $\tilde{\Phi}(u)=u^{2}$, $\Phi(u)=\infty$. It is an open question whether or when the second inequality can bo stirict (see [3]).

6. By making a wild induction from the preceding examples one could guess oux result to bo sharp if $\Phi(\dot{u})<u^{p}$ for all $p>1$, but we can offer no reasons for such a belief but the desire to have an index so easily computiablo. As the last example of this easiness, we give the result for the "xectangles in a Ountor set of directions" basis: $\Phi(u) \simeq O u^{1+}$ where $8=. \log 2 / \log 3$ for the usual Cantor set.

\section{Roferences}

[1] A. Cordoba, $8 \times t \times \varphi(8, t)$, Mittag-Leffler Inst. report $n^{\circ} 8$ (1978).

[2] J. El Telou, Recouvrement du tore T'a par des ouverts aléatoires, These. Unir. Paris (Orway) (1878).

[3] M. Guzmán, Differentiation' of integrats in $\boldsymbol{R}^{n}$, Lecture Notes $481,1975$. 
[4] B. Jessen, J. Marcinkiewicz, A. Zygmund, Nate on thd differentiability of multiple integrals, Fund. Math. 25 (1935).

[5] J.M. Marstrand, A counter-example in the theary of strong differentiation, Bull. London Math. Soc. 9 (1977), 209-211.

[6] R. Moriyón, El halo en la teoría de diferenciación de integrales, Tesis. Univ. Complutense, Madrid 1978.

[7] A. Nagel, E. Stein, S. Wainger, Differentiation in lacunary direotions, Proc. Nat. Acad. Sci. U. S. A. 75, 3 (1978).

[8] J. Stro in berg, Maximal funetions for rectangles with given directions, MittagLeffler Institute (1977).

FAOULTAD DW MATEMATIOAS, MADRID
UNIVERSIDAD COMPLUTHSE, MADR 